\title{
INOVAÇÃO, EMPREENDEDORISMO E ECONOMIA PRATEADA NA PANDEMIA DA COVID-19: NOVOS RUMOS DO MERCADO
}

\author{
Luciana Mitsue Sakano Niwa' \\ ORCID: 0000-0002-9342-7454 \\ Larissa Sapucaia F. Esteves" \\ ORCID: 0000-0003-3489-2599 \\ Daniela Garcia Damaceno" \\ ORCID: 0000-0001-8656-009X
}

Sergio Wether Duque Estrada"'

ORCID: 0000-0003-2175-199X

Suely Itsuko Ciosak'

ORCID: 0000-0001-5884-2524

'Universidade de São Paulo.

São Paulo, São Paulo, Brasil.

"Universidade do Oeste Paulista. Presidente Prudente, São Paulo, Brasil.

"'Representante do AGING 2.0 para a América do Sul. São Paulo, São Paulo, Brasil.

Autor Correspondente: Luciana Mitsue Sakano Niwa E-mail: lucianamsn@usp.br

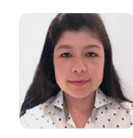

Como citar:

Niwa LMS, Esteves LSF, Damaceno DG, Estrada SWD, Ciosak SI. Inovação, empreendedorismo e economia prateada na pandemia da covid-19: novos rumos do mercado. In: Santana RF (Org.). Enfermagem gerontológica no cuidado do idoso em tempos da COVID 19. Brasilia, DF: Editora ABen; 2021. 171 p.

(Serie Enfermagem e Pandemias, 5). https://doi.org/10.51234/aben.21.e05.c07

\section{INTRODUÇÃO}

A população brasileira vivenciou nas últimas décadas profundas mudanças que trouxeram impactos em diversos aspectos em seu perfil populacional. Entre os anos 1950-1970, com a redução da mortalidade infantil e alta fecundidade após Segunda Guerra Mundial, o país vivenciou o período chamado de Baby Boom. Contudo, o período que durou apenas duas décadas, foi seguido de uma queda acelerada da taxa de fecundidade, que associada a maiores expectativas de vida pela evolução tecnológica e por melhorias no saneamento básico, alterou todo processo saúde-doença prolongando a vida, e sendo assim responsável por uma transição demográfica acelerada no país ${ }^{(1)}$.

Os dados demográficos evidenciam o crescimento acentuado dessa parcela da população, cujos números da Fundação do Sistema Estadual de Análise de Dados (FUNDAÇÃO SEADE, 2020), mostram que no Estado de São Paulo as pessoas com mais de 60 anos já somam $14,86 \%$ da população geral. A projeção para 2050 é que o percentual de pessoas com $60+$ anos chegue a $29,8 \%$. Essa perspectiva mostra que em alguns estados brasileiros a quantidade de pessoas idosas será maior que a projeção para o Brasil, onde o percentual alcançará quase $22 \%$ em $2050^{(2,3)}$.

Embora se reconheça que o envelhecimento populacional é uma conquista social do século $X X$, sabe-se que ele traz desafios para a sociedade e para as políticas públicas. Entre eles, um dos mais importantes é assegurar que o desenvolvimento econômico e social ocorra continuamente, assegurando a manutenção da dignidade humana e equidade entre os grupos etários, garantindo o acesso a recursos, direitos e responsabilidades sociais ${ }^{(4)}$.

A nova geração de gestores públicos enfrentará um desafio diferente nos próximos anos: ao invés de investir em creches, provavelmente, a demanda maior será por novos centros de acolhimento ou de convivência para 
idosos ${ }^{(5)}$. Dessa forma, o ritmo de crescimento tecnológico que ocorre no mundo todo, oferece oportunidades de avanços para a melhora da qualidade de vida dessa população, uma vez que pode trazer eficiência para a vida cotidiana por meio de assistência funcional, monitoramento de segurança, consultas de saúde de forma remota e inclusão social ${ }^{(6)}$.

Em muitos países desenvolvidos a população de pessoas idosas tem se tornado a alavanca do consumo global, pois é o grupo etário que mais cresce e dos que mais consome. A estimativa é que eles deverão gerar $51 \%$ do crescimento do consumo urbano ou US\$4.4 trilhões até $2030^{(7)}$. No Brasil, os idosos movimentaram uma renda de 964 bilhões em consumo em 2018 e estão se transformando em alvos de interesse no mercado consumidor, inclusive da indústria do turismo ${ }^{(8)}$. Embora haja desafios ao lidar com os impactos de uma gestão macroeconômica, política e social, as demandas dessa população trazem um novo campo de desenvolvimento no mercado ${ }^{(9)}$.

Contudo, a pandemia da Covid-19 trouxe um cenário de incertezas para o mercado financeiro e principalmente para as iniciativas voltadas para os idosos. A Covid-19 é a doença infecciosa causada pelo vírus Sars-Cov-2, cujo principal grupo de risco são as pessoas idosas pela diminuição da eficácia do sistema imune e pela presença prévia de doenças crônicas não transmissíveis. Os idosos também dilatam as taxas de mortalidade em todo o mundo. Como ações de proteção e contenção da disseminação do vírus, medidas sanitárias e o distanciamento social foram estabelecidas e até impostas, particularmente, para a população idosa.

Destarte, frente ao contexto da pandemia da Covid-19 e os impactos do distanciamento para a pessoa idosa, o presente artigo tem o intuito de refletir acerca da economia voltada a esse segmento populacional e os desafios e oportunidades empreendedoras no cenário atual e pós-pandemia.

\section{OBJETIVO}

Refletir sobre a "Economia Prateada" e as ações empreendedoras para a pessoa idosa no cenário atual e pós pandemia da Covid-19

\section{MÉTODO}

Estudo teórico-reflexivo baseado em literatura nacional e internacional e nos relatos de experiência dos autores. O texto tece considerações sobre:

- Perfil da população idosa;

- Gerontotecnologia e empreendedorismo na longevidade;

- Os desafios do empreendedorismo para a pessoa idosa no país e no contexto da pandemia;

- Cenários de oportunidades direcionadas para população idosa no contexto atual e pós-pandêmico da Covid-19.

\section{RESULTADOS}

\section{O perfil da população idosa brasileira e a pandemia do coronavírus}

Um aspecto fundamental para o desenvolvimento de projetos e políticas para os idosos, é reconhecer, em primeiro lugar, que se trata de uma população heterogênea. Há idosos com excelentes condições de saúde e adeptos de atividades físicas, enquanto há outros com a saúde debilitada e restrição de mobilidade. Há os com alta taxa de escolaridade formal e inúmeros outros analfabetos. Em um país com grande desigualdade socioeconômica, encontramos idosos que passam por situações financeiras difíceis, até para morar e comer, e são poucos os que vivem com tranquilidade decorrente das economias poupadas ao longo da vida ${ }^{(5)}$.

Em tempos de pandemia, tais diferenças mostraram-se ainda mais evidentes e repercutem em um cenário de incertezas para o mercado financeiro, principalmente, para as iniciativas voltadas para os idosos. 
Dentro dos domicílios a população idosa representa 19,3\% das pessoas de referência ou chefes de família, corroborando com a ideia deles como o amparo da família - o que dificulta, muitas vezes, a política de distanciamento social na pandemia da COVID-19. A respeito da escolaridade, a taxa de analfabetos entre essa faixa etária chega a $30 \%$, sendo que $16,6 \%$ possuem entre 1 e 3 anos de escolaridade ${ }^{(10)}$.

Nessa faixa etária encontram-se as menores desigualdades de distribuição de renda se comparada à população total. Acerca da ocupação, 28,7\% das pessoas inativas em 2018 eram idosas, sendo mais comum encontrá-las nos setores da economia da agricultura e pecuária $(6,62 \%)$ e da alimentação $(4,12 \%)$, sendo estes os principais afetados no cenário da pandemia ${ }^{(10)}$.

Tratá-los como um conjunto uniforme é um equívoco que não deve ser cometido pelos gestores públicos e empresariais. Tais diferenças mostram que é necessário avançar com ações para além das áreas da saúde e do social, embora estas ainda sejam as maiores demandas. A população idosa requer ações, projetos e políticas em áreas como educação, cultura, esporte, lazer, desenvolvimento econômico, ciência e tecnologia, habitação, transporte, segurança, entre outras. A lógica futura necessita de projetos intersetoriais, que envolvam e integrem várias áreas (5).

Sendo assim, atender às necessidades de uma sociedade que está envelhecendo significa não apenas fornecer cuidados básicos de saúde, mas executar ações que possam melhorar a qualidade de vida dessa parcela da população. A tecnologia da comunicação e informação pode oferecer suporte de vida para os idosos, aumentando sua independência, melhorando a mobilidade física e seus níveis de atividade, e aumentando as conexões sociais e a segurança. Já é possível aplicar sistemas de telemonitoramento e sistemas de E-saúde, como teleconsultas e telereabilitação, que atualmente estão sendo amplamente utilizados no sentido de fornecer meios para realizar o distanciamento social, necessário em tempos de pandemia. Além disso, as tecnologias têm aumentado a possibilidade de suporte social para idosos que vivem de forma independente na comunidade em tempos de COVID-19 ${ }^{(6,11)}$.

Entretanto, é válido ressaltar que em 2018, o acesso dessa população à internet no Brasil, era de 38,7\%. Assim, embora essa taxa tenha apresentado um crescimento de 24\% entre 2017-2018, é um dado importante a ser considerado pelo mercado ao se pensar no desenvolvimento de produtos voltados a esse grupo ${ }^{(10)}$. Mas observa-se que o uso da tecnologia pelos idosos vêm aumentando significativamente nos últimos cinco anos no Brasil e considerando a escolaridade e condição socioeconômica do país, ainda há muito que se realizar para evitar o "apartheid digital" e promover a "literacia tecnológica" (12).

\section{Gerontotecnologia e empreendedorismo na longevidade}

A "Economia da Longevidade" ou "Economia Prateada" é uma forma de organização do sistema econômico e financeiro, baseada na gerontotecnologia, isto é, no uso de intervenções de bases científicas e tecnológicas voltadas às necessidades de pessoas idosas, ao processo de envelhecer, reduzindo os impactos considerados negativos do envelhecimento populacional. Este termo ganhou força na União Europeia, a partir de 2007 com o desenvolvimento de reformas voltadas a este segmento de mercado como um conjunto, incluindo tecnologia da informação, telecomunicações, setor financeiro, habitação, transporte, energia, turismo, cultura, infraestrutura, serviços locais e de cuidados de longa duração. Dessa forma, a Economia Prateada, não é um setor independente, mas sim um mercado transversal envolvendo vários setores industriais ${ }^{(13)}$.

Já no Brasil, é definida como um conjunto de medidas estratégicas propostas pelo Estado com a finalidade de estimular pesquisadores e o setor privado a explorar oportunidades fomentadas pelo processo de enveIhecimento por meio da inovação, promovendo a adaptação da economia à nova dinâmica populacional com vistas ao desenvolvimento econômico ${ }^{(9)}$.

O termo Gerontotecnologia também tem sido utilizado para determinar produto, processo, estratégias, serviços e/ou conhecimentos que tem a finalidade de entregar ações cuidativas educacionais para a pessoa idosa e seus familiares/cuidadores por meio da construção coletiva que valoriza as relações, interações e retroações dos envolvidos por meio do conhecimento inter-multi-transdisciplinar ${ }^{(14)}$. 
A gerontotecnologia se mostra, portanto, como seu elemento-chave. Desde 1991, segue cinco diretrizes principais: (1) prevenção de problemas; (2) aumento da capacidade para lidar sozinho com problemas sem necessidades de mudanças no ambiente ou desenvolvimento; (3) compensação da perda de opção quando for possível oferecer meios de facilitar situações; (4) prestação de cuidados somente quando necessário, (5) estudo e melhoria de designs existentes ${ }^{(13)}$.

O acesso a dispositivos tecnológicos e à internet se fazem necessários para o desenvolvimento da Economia da Longevidade pautada na Gerontotecnologia. Nos estados Unidos cerca de $80 \%$ da população de pessoas mais velhas possuem um smartphone e desses cerca de mais da metade $(46,3 \%)$ indicam que podem utilizá-los de forma eficaz. Um tablet era propriedade de $74,3 \%$ dos idosos e $52,8 \%$ afirmaram possuir e utilizar relógios inteligentes como forma de monitoramento de atividades da vida cotidiana ${ }^{(15)}$.

Dispositivos que garantam a interação, saúde mental, mobilidade, lazer, atenção à saúde foram identificados por idosos como sendo aqueles de maior utilidade, pois ajudam na qualidade de vida. A interação social por meio de mensagens curtas por dispositivos de comunicação foi considerada pelos idosos como a função tecnológica mais popular para manter conexões com familiares. Em tempos de pandemia, se mostrou uma ferramenta de extrema importância para garantir o distanciamento social, sem fomentar o isolamento ${ }^{(6)}$.

Entretanto, as interfaces tecnológicas para os idosos devem considerar as limitações funcionais decorrentes do processo fisiológico do envelhecimento. Alguns princípios devem ser seguidos para empreender nesta vertente, dentre elas a interface deve ter complexidade intuitiva, estrutura clara, informações consistentes, feedback instantâneo, fornecimento de ajuda, interação otimizada e personalização ${ }^{(6)}$.

No que tange o empreendedorismo, existem diferentes níveis e dimensões a serem exploradas quando se vislumbra como clientes as pessoas idosas, contudo há cinco principais áreas: (1) saúde e dignidade; (2) habitação e vida cotidiana; (3) mobilidade e transporte; (4) comunicação e gestão; (5) trabalho e lazer; (6) vida financeira. Desse modo, diante das oportunidades, considerando conhecimento e afinidade ao grupo de pessoas idosas, muitas propostas empreendedoras começaram a tomar forma no Brasil. O empreendedorismo da população brasileira pode ser resultado da transformação cultural gerada pela troca de procura por emprego para a abertura do próprio negócio, gerando as bases para a criação de Startups ou de Seniortechs, como vêm sendo chamadas.

Não existe um consenso sobre definição de Startup, sendo a mais aceita a proposta por BLANK E DORF (2012): "é um modelo de negócio interino, cujo objetivo é alcançar um modelo de negócio escalável e repetitivo"(16). Desde que o conceito de startup tomou forma no Brasil, a quantidade de pessoas que se dedicam ao ramo só tem aumentado nos diversos segmentos ${ }^{(17)}$. Em relação às startups para pessoas idosas, o evento Startup Search computou 72 iniciativas em 2018 com fins lucrativos, e 93 em 2019, um crescimento de 29,1\%, ganhando visibilidade e atraindo atenção crescente do mercado de investidores ${ }^{(18)}$.

\section{Os desafios do empreendedorismo para a pessoa idosa no país e no contexto da pandemia}

Para além dos desafios encontrados durante a pandemia no Brasil, as questões burocráticas relacionadas às pesquisas acadêmicas mostram-se como um desafio importante para o empreendedorismo nacional. $\mathrm{Na}$ área da robótica assistiva, por exemplo, os impositivos legais são enormes. Os robôs importados, fundamentais para a pesquisa, são sobretaxados como brinquedos e peças que em outros países têm baixo custo para os pesquisadores, no Brasil são raras e adquiridas a preços muito elevados, sofrendo impactos principalmente da carga tributária ${ }^{(9)}$.

A pandemia da Covid-19 trouxe uma retração das startups voltadas aos idosos pois a expressiva maioria estava iniciando e houve uma redução no volume de capital de risco disponível no mercado; esse fator, somado à incapacidade de atender imediatamente a demanda de algum contato presencial, à falta de conhecimento/intimidade dos usuários finais com os serviços e produtos (dependência de terceiros, familiares e 
cuidadores);à redução drástica de liquidez levou a retração de várias iniciativas (corte de custos: investimento/ pessoal/tecnologia e; investidores retraídos).

O distanciamento do convívio pessoal com a família e a sociedade fez da tecnologia digital, fundamental para a comunicação entre as pessoas, para continuidade do trabalho em casa, para compras essenciais, pagamento de despesas, entre outros. Os idosos utilizavam a tecnologia digital, muitas vezes, com auxílio de familiares e amigos. Diante do distanciamento, muitos ficaram em suas casas sozinhos ou acompanhados de outros idosos e tiveram que aprender a usar outras funcionalidades do celular, bem como acessar plataformas para comprar insumos do dia a dia.

Contudo, quando o assunto é a população idosa e tecnologia digital a principal barreira é o idadismo ou ageísmo. Assim, embora se reconheça o potencial de aproximação social proporcionado pela tecnologia, a falta de acessibilidade das ferramentas tecnológicas aos idosos e a visão preconceituosa e estereotipada sobre esta parcela populacional com sua consequente ridicularização, dificultam o acesso dessas pessoas às tecnologias, sendo necessário o desenvolvimento de estratégias que atendam suas necessidades e particularidades ${ }^{(19)}$. Nesta vertente pode-se observar uma janela de oportunidade para quem quer empreender com tutoriais de aprendizagem de ferramentas tecnológicas para a população idosa.

\section{Cenários de oportunidades}

A população idosa está cada vez mais conectada no uso da internet em mídias sociais e e-commerce. A adaptação ao meio tecnológico é crescente e aumenta cada vez mais em tempos de pandemia e distanciamento. Os idosos possuem smartphone e são plenamente atendidos por Whatsapp além de acessarem redes sociais como Instagram e Facebook.

As iniciativas digitais voltadas para os idosos precisam começar a ter linguagem, design e modelos intuitivos específicos para essa população. Confiança e segurança com a presença de todos os dados da startup/seniortechs como endereço, CNPJ, telefone, certificados de segurança e diversas formas de pagamento incluindo boleto e depósito bancário são formas de assegurar o consumidor idoso. Outra forma de aumentar a confiança do consumidor é ter canal de comunicação para atendimento, reforçando a humanização. Além do layout simples, intuitivo, fácil e, principalmente, legível onde é possível a leitura sem necessitar aproximar a tela.

Algumas iniciativas de sucesso voltadas para atender os idosos são as plataformas para busca de cuidadores e profissionais de saúde sem intermediação, os dispositivos de monitoramento de quedas, auxílio no transporte de idosos com limitações, manutenção neurológica e cognitiva por meio de jogos de celular adaptados aos $60+$ e as inúmeras atividades de tele-consulta/atendimento.

Dessa forma, é necessário que essas empresas se adaptem à realidade desta população crescente, reconhecendo suas principais demandas, dificuldades, buscando alcançar soluções que contribuam com a manutenção da autonomia, independência e envelhecimento ativo. Neste contexto, o papel das Startups / Seniortechs, pesquisadores e empresas é desenvolver estratégias e produtos que possibilitem a autonomia e funcionalidade dos idosos, bem como seu protagonismo na sociedade e acesso a rede social e de apoio por meio de tecnologias digitais, adaptação das habitações, das infraestruturas, e dos serviços.

\section{CONSIDERAÇÕES FINAIS}

A pandemia da Covid-19 nos convida a ressignificar e reinventar o novo normal. O momento singular em que vivemos pode ser uma grande inspiração e oportunidades para empreender em atividades e negócios voltados para os idosos.

Vale lembrar que muitos hábitos desenvolvidos pelos idosos nesta pandemia, podem se perpetuar, através de novos hábitos, facilidades e principalmente o exercício do raciocínio e memória, que deve ser valorizado, melhorado e explorado, para facilitar alguns enfrentamentos decorrentes do envelhecimento. 
Pessoas idosas estão quebrando paradigmas e adentrando a era tecnológica. Cabe aos pesquisadores, profissionais, startups e empresários abdicarem de seus preconceitos, olharem o copo como cheio e ousarem mergulhar nas águas da Economia Prateada.

\section{AGRADECIMENTO}

\section{ABEn e DCEG nacional.}

\section{REFERÊNCIAS}

1. Miranda GMD, Mendes ACG, Silva ALA. Desafios das políticas públicas no cenário de transição demográfica e mudanças sociais no Brasil. Interface (Botucatu). 2017;21(61):309-20. https://doi.org/10.1590/1807-57622016.0136

2. Fundação Sistema Estadual de Análise de dados (SEADE). População com 60 anos e mais [Internet]. São Paulo. 2020[cited 2020 Aug 17]. Available from: https://perfil.seade.gov.br/

3. Instituto Brasileiro de Geografia e Estatística (IBGE). Projeções da população: Brasil e unidades da federação. Revisão de 2018 [Internet]. Rio de Janeiro, 2018[cited 2020 Aug 17]. 56p. Available from: https://www.ibge.gov.br/apps/populacao/ projecao/ acesso em 15/08/2020.

4. Camarano AA. Os marcos internacionais e as políticas brasileiras em prol da população idosa. In. Souza AM, Miranda P (Eds). Brasil em desenvolvimento 2015: Estado, planejamento e políticas públicas [Internet]. Brasília: Ipea; 2015 [cited 2020 Aug 17]. p 239-265

5. Burgos F. Os idosos na agenda governamental [Internet]. GV-Executivo. 2020[cited 2020 Aug 17];19(1):36-8. Available from: https://rae.fgv.br/sites/rae.fgv.br/files/gv_191art3.pdf

6. Lin CJ, Ho SH. Prediction of the use of mobile device interfaces in the progressive aging process with the model of Fitts' law. J Biomedic Inform. 2020. p.103457. https://doi.org/10.1016/j.jbi.2020.103457

7. Bughin J, Woetzel J. Navigating a world of disruption [Internet]. Mckinsey Global Institute, Switzerland, 2019[cited 2020 Aug 17]. Available from: https://www.mckinsey.com/featured-insights/innovation-and-growth/ navigating-a-world-of-disruption

8. Henkel M. Idosos são o novo filão da indústria e serviços [Internet]. Jornal do Comércio [Internet]. Porto Alegre. 2017[cited 2020 Aug 17]. Available from: https://www.jornaldocomercio.com/_conteudo/2017/10/economia/591271-idosos-saonovo-filao-da-industria-e-servicos.html

9. Felix JS. Economia da Longevidade, Gerontecnologia e o complexo econômico-industrial da saúde no Brasil: uma leitura novo-desenvolvimentista. Rev Kairós: Gerontol. 2018;21(1):107-30. https://doi.org/10.23925/2176-901X.2018v21i1p107-130

10. Instituto Brasileiro de Geografia e Estatística (IBGE). PNAD contínua TIC: estatísticas sociais [Internet]. 2017[cited 2020 Aug 17]. Available from: https://agenciadenoticias.ibge.gov.br/agencia-sala-de-imprensa/2013-agencia-de-noticias/ releases/23445-pnad-continua-tic-2017-internet-chega-a-tres-em-cada-quatro-domicilios-do-pais

11. Hollander JE, Carr BG. Virtually perfect? Telemedicine for COVID-19. N England J Med. 2020;382(18):1679-81. https://doi. org/10.1056/NEJMp2003539

12. Rossi RC, Valentim MLP. Globalização de serviços para a cidadania: uma revisão bibliográfica sobre a infoexclusão no Brasil. Pág A\&B: Arq Bib [Internet]. 2020[cited 2020 Aug 17]. p. 33-45. Available from: https://ojs.letras.up.pt/index.php/ paginasaeb/article/view/6835/7797

13. Klimczuk A. Supporting the development of gerontechnology as part of silver economy building. Ad Alta: J Interdiscip Res [Internet]. 2012[cited 2020 Aug 17];2(2):52-6. Available from: https://ssrn.com/abstract=2203522

14. Ilha S, Santos SSC, Backes DS, Barros EJL, Pelzer MT, Costenaro RGS. (Geronto)tecnologia cuidativo-educacional complexa para pessoas idosas/famílias com a doença de Alzheimer. Rev Bras Enferm. 2017;70(4):726-32. https://doi. org/10.1590/0034-7167-2016-0687

15. Arthanat $\mathrm{S}$, Chang $\mathrm{H}$, Wilcox J. Determinants of information communication and smart home automation technology adoption for aging-in-place. J Enabling Technol. 2020. https://doi.org/10.1108/JET-11-2019-0050

16. Blank SG, Dorf B. The startup owner's manual: the step-by-step guide for building a great company. K\&S Ranch Publishing Division; 2012. 
17. Pires JG. Alguns insights em startups: um novo paradigma para a tríplice aliança ciência, tecnologia e inovação. Rev Gestão Saúde. 2020;11(1). https://doi.org/10.26512/gs.v10i3.26410

18. Ativen. Catálogo Seniortech: startup search, feira da longevidade [Internet]. 2019[cited 2020 Aug 17]. Available from: https://www.ativen.com.br/catalagoseniortech

19. Hammerschmidt KSA, Santana RF. Saúde do idoso em tempos de pandemia Covid-19. Cogitare Enferm. 2020;25. https:// doi.org/10.5380/ce.v25i0.72849 